\title{
An Italian pediatric case series of Maldive-imported dengue fever
}

\author{
Arianna Dondi, ${ }^{1}$ Francesca Gottardi, ${ }^{1}$ Marianna Fabi, ${ }^{1}$ Luciano Attard, ${ }^{2}$ Giada Rossini, ${ }^{3}$ \\ Maria Carla Re, ${ }^{3}$ Marcello Lanari ${ }^{1}$ \\ ${ }^{1}$ Pediatric Emergency Unit, Department of Medical and Surgical Sciences; ${ }^{2}$ Infectious Diseases Unit, \\ Department of Medical and Surgical Sciences; ${ }^{3}$ Unit of Microbiology, Regional Reference Centre for \\ Microbiological Emergencies (CRREM), Sant'Orsola-Malpighi Hospital, Bologna, Italy
}

\begin{abstract}
Dengue is a mosquito-borne disease frequently imported in Europe, where autochthonous outbreaks are potential since recent spreading of the vector. Primary infections usually produce a selflimited febrile syndrome. Secondary infections with different serotype, especially in children, may lead to a severe shock syndrome with plasma leakage and hemorrhagic features. We report a case series of 4 children infected with viral serotype 3 during a journey to the Maldive Islands, who developed symptoms after returning to Italy. Once diagnosed, they were admitted to hospital, followed with clinical and laboratory monitoring and treated supportively; they developed no complications. Dengue endemic areas are popular touristic destinations. Vector eradication may be incomplete where tourist resorts share water tanks with local dwellings. Diagnostic tests should be available in our hospitals for early recognition of cases. Awareness of a primary infection may help prevent re-exposure, avoiding a reinfection with a potentially severe clinical course.
\end{abstract}

Correspondence: Arianna Dondi, Pediatric Emergency Unit, Department of Medical and Surgical Sciences, Sant'Orsola-Malpighi Hospital, University of Bologna, via Massarenti 11,

40138 Bologna, Italy.

Tel.: +39.051.2143012.

E-mail: arianna.dondi@aosp.bo.it

Key words: Dengue; Pediatric; Infectious disease; Arbovirus; Tropical disease.

Contributions: AD: conception and design of the work; data analysis and interpretation; revised the work critically for important intellectual content. FG: conception of the work; data acquisition, analysis and interpretation; drafting of the work. MF: conception of the work; revised the work critically for important intellectual content. LA, GR, MCR, ML: data interpretation; revised the work critically for important intellectual content. All authors approved the final version of the manuscript.

Conflict of interest: the authors declare no potential conflict of interest.

Funding: none.

Received for publication: 20 October 2018.

Revision received: 7 December 2018.

Accepted for publication: 12 December 2018.

This work is licensed under a Creative Commons Attribution 4.0 License (by-nc 4.0).

(C) Copyright A. Dondi et al., 2019

Licensee PAGEPress, Italy

Emergency Care Journal 2019; 15:7892

doi:10.4081/ecj.2019.7892

\section{Introduction}

Dengue is caused by one of four single-stranded RNA viruses (serotype 1-4) of the Flaviviridae family, transmitted between humans by Aedes mosquitoes. The vector is widely distributed across tropical and subtropical regions, many of which are touristic destinations, and is one of the most frequently imported diseases in Europe. ${ }^{1}$ Infection is characterized by an initial febrile phase lasting 3-7 days, presenting with elevated temperature $\left(\geq 38.5^{\circ} \mathrm{C}\right)$, headache, myalgia, joint pain, gastro-enteric symptoms and transient macular rash. Children may develop very high temperatures but are often less symptomatic than adults. Thrombocytopenia and leukopenia are typical laboratory findings. ${ }^{2}$

Most patients recover, but a small part, frequently children and young adults, during defervescence develop a life-threatening syndrome characterized by plasma leakage, hemoconcentration, pleural and abdominal effusion, bleeding, shock, and organ impairment. This phase characterizes severe disease and is often caused by secondary infections by different serotypes. Lack of long-term cross-immunity among the four virus types allows for multiple sequential infections. The widely accepted explanation for the development of severe disease is the antibody-dependent enhancement model, which suggests that cross-reactive non-neutralizing antibodies increase viral pathogenicity. ${ }^{3}$

In 2009, WHO published a revised classification which distinguished between severe and non-severe Dengue. ${ }^{4}$ Severe Dengue is also identified as Dengue shock syndrome. Non-severe Dengue includes probable Dengue, Dengue without warning signs (previously Dengue fever) and Dengue with warning signs (previously Dengue hemorrhagic fever).

Warning signs designated as predictors of severe disease are: persistent vomiting, increasingly severe abdominal pain, tender hepatomegaly, a high or increasing hematocrit $\mathrm{t}$ with a rapid decrease in the platelet count, serosal effusions, mucosal bleeding, and lethargy or restlessness. Their presence warrants hospital admission and close clinical and hematological monitoring (daily complete blood count is suggested to monitor hematocrit and platelet value) to rapidly identify patients who need supportive treatments.

Vascular leakage and shock are more frequent and severe in children, while bleeding manifestations and organ involvement are more common in adults. The laboratory diagnosis of Dengue is established directly by detection of viral components in serum or indirectly by serologic means. Treatment is only supportive, in the absence of effective antiviral agents, and is based on careful fluid management.

As immunization is not yet available, the basis of prevention is still vector control, through direct targeting of mosquitoes and removal of their breeding sites.

Imported infections require prompt diagnosis to reduce the risk of autochthonous outbreaks and appropriate monitoring and management to prevent clinical complications, especially in children. ${ }^{2}$ 


\section{Case Reports}

We report the clinical course of Dengue serotype 3 infection in a small cluster of children (age 7-12 years), back from a journey to an endemic area (Republic of Maldives). The patients were two couples of siblings who had spent two weeks with their families in a small touristic structure on an island where local dwellings coexisted.

In April 2018, 5-7 days after being back to Italy, they came to the Pediatric Emergency Department (PED) of Sant'OrsolaMalpighi Hospital (Bologna, Italy) for a fever of recent onset. In all cases a tropical fever diagnostic panel was performed, including Dengue, Chikungunya and Zika tests. The diagnosis was promptly performed by NS1 antigen detection (enzyme immuno assay, Platelia Dengue NS1 AG Kit; BioRad, Segrate, Italy) with results available in few hours and nucleic acid detection (multiple Real Time PCR for Dengue RNA, available in 24 hours and capable of detecting serotype). In all cases, serotype DENV-3 was identified. Dengue-specific IgM and IgG (Euroimmun, Lübeck, Germany, immunofluorescent assay) were also tested but not found in serum as expected in the early stages of infection. Patients' 1 and 2 father was also diagnosed with DENV-3 infection.

All patients were admitted in order to provide close clinical and laboratory monitoring. Hospital admission also allowed the prevention of any contact with possible mosquito vectors during the viremia. In fact, even if the season was regarded as safe at our latitude for circulation of Aedes mosquitos, isolation during the acute phase of illness was considered safer. Moreover, children benefited of supportive treatment during the febrile phase.

Blood count checks showed progressive development of lymphopenia and thrombocytopenia in all four patients. These features are typical of Dengue infection and in our cases were self-limited with similar timing of nadir (Figure 1).

All patients were discharged at the end of the febrile phase, after complete clinical stabilization.

After discharge, blood tests were repeated again 10-12 days after the onset of symptoms and, in all cases, seroconversion was confirmed by the appearance of Dengue-specific IgM and IgG. Here we describe the four cases in detail.

\section{Case \#1}

11-year-old girl referred to the PED after 3 days of fever, nausea and fatigue. On physical examination, a temperature of $39^{\circ} \mathrm{C}$ and mild dehydration were detected. In addition, a transient macular rash was reported.

Upon admission, venous access was obtained, and intravenous fluid support was performed until an appropriate oral hydration was possible. Fever lasted 5 days, with daily temperatures up to $40^{\circ} \mathrm{C}$, associated with joint/bone pain without signs of arthritis. After the febrile phase, a mild erythematous itchy rash appeared. Recovery was rapid and complete.

Blood count monitoring showed leukopenia (WBC 1600, L 650 cells $/ \mathrm{mL}, \mathrm{N} 950$ cells $/ \mathrm{mL}$ on the $4^{\text {th }}$ day of fever) and mild thrombocytopenia ( 130.000 platelets $/ \mathrm{mL}$ on $6^{\text {th }}$ day of fever). Hematocrit remained within normal range and no other altered findings were detected. A mild, transient increase in aminotransferases was noted during the recovery phase.

\section{Case \#2}

7 -year-old boy admitted at the same time of his sister (patient 1) 24 hours after the onset of fever, myalgia, joint pain and fatigue.

Physical examination was normal except for temperature $39^{\circ} \mathrm{C}$. During clinical monitoring, fever continued with tempera- tures up to $40^{\circ} \mathrm{C}$ and was treated with acetaminophen. On day 2 , he developed nausea and mild diarrhea, so he was supported with intravenous fluids. Daily blood count showed progressive development of lymphopenia (nadir WBC $1760, \mathrm{~L} 400$ cells $/ \mathrm{mL}$, on the $4^{\text {th }}$ day of fever) and mild thrombocytopenia. On day 5 temperature decreased permanently and recovery was complete.

\section{Case \#3}

12-year-old boy referred to our PED with a 24-hour history of fever and headache with poor response to acetaminophen. On physical examination he had a temperature of $39^{\circ} \mathrm{C}$ and a diffuse macular rash. He reported nausea and anorexia. He was admitted, and a venous access was obtained for intravenous fluid support and treatment with acetaminophen.

Daily blood count showed progressive development of lymphopenia (nadir 530 cells $/ \mathrm{mL}$ on day 5) and mild thrombocytopenia (nadir 130.000 platelets $/ \mathrm{mL}$ on day 6 ).

On day 4 patient reported severe asthenia and occurrence of watery diarrhea. Hematocrit was not increased but platelet count severely fell since the previous day. Coagulation check showed mild prolongation of aPTT (2.01). Chemical panel also showed hyperferritinemia $(1938 \mathrm{ng} / \mathrm{dL})$ and rise of aminotransferases (AST $183 \mathrm{UI} / \mathrm{L}$, ALT $188 \mathrm{UI} / \mathrm{L}$ ).

A mild decrease in arterial blood pressure $(90 / 55 \mathrm{mmHg})$ and mild hypoglycemia $(60 \mathrm{mg} / \mathrm{dL})$ due to anorexia were detected and intravenous fluid support was potentiated and corrected with glucose.

Clinical conditions progressively improved. On day 5 the fever disappeared and a severe erythematosus macular rash with areas of spare skin appeared on the legs.

\section{Case \#4}

5 -year-old boy, patient 3's brother, admitted at the very beginning of fever, which rose up to $40^{\circ} \mathrm{C}$ and continued for the following days associated with frontal headache, nausea and anorexia.

Daily blood count showed progressive lymphopenia (nadir 450 cells $/ \mathrm{mL}$ on day 4) and moderate thrombocytopenia (nadir 50.000 platelets/mL on day 5). He also presented hyperferritinemia (1679 $\mathrm{ng} / \mathrm{dL}$ ), a rise of aminotransferases (AST $142 \mathrm{UI} / \mathrm{L}$, ALT $89 \mathrm{UI} / \mathrm{L}$ ) and a mild prolongation of aPTT. Complete clinical recovery on day 6 (Table 1).

\section{Discussion}

In European databases ${ }^{5}$ Dengue is included among the most important travel-related infections. Moreover, the International Geo-Sentinel Surveillance Network reports that Dengue-related infections are now the most common cause of fever among travelers, overcoming malaria and gastrointestinal infections. ${ }^{6}$ A recent study about imported Arbovirus infections in Italy reports Dengue as the most frequently diagnosed disease during the period July 2014-October 2015 . $^{7}$

Therefore, Dengue should be considered in any patient presenting with fever onset within 14 days after a trip to the tropics and subtropics.

In the Republic of Maldive, Dengue fever has been endemic since 2004 with seasonal outbreaks. ${ }^{8}$ Nevertheless, imported cases from Maldives are rare, thanks to a systematic control of the mosquito-vector which is strictly supervised by tour-operators in the whole archipelago. However, in the last years, many new guesthouses managed by single owners or families were opened. Where these touristic structures share water tanks with local dwellings, 
the eradication of vectors can be incomplete, possibly leading to small primary infection outbreaks among tourists, as in the case series described.

Given the speed of air travel, even diseases with short viremia like Dengue can be exported to naive areas within 24 hours. Small autochthonous clusters occurred in Southern France and in Croatia in 2010, and a major outbreak involving more than 2000 persons occurred in Madeira/Portugal in 2012. ${ }^{9}$

Recently, the dramatic global distribution of Aedes albopictus (known as "tiger-mosquito") has caused concern over the possibility of Dengue transmission as a competent vector. Although these mosquitos have a lower competence than Aedes aegypti, autochthonous infections can be established by travelers returning from endemic zones to areas with high $A$. albopictus density, like many Italian regions. ${ }^{10}$ It was in this way that Chikungunya virus was introduced to Northern Italy in $2007 .{ }^{10}$ Thus, epidemiological and laboratory surveillance is crucial to rapidly identify imported cases in order to introduce measures to reduce risks for public health.

In Emilia Romagna (Northern Italy), an active regional surveillance program was established in 2007 to guarantee early diagnosis of Dengue infections in individuals returning from high-risk areas. ${ }^{11}$ In 2010, the frequency of Dengue infection was estimated $20.5 \%$ in febrile patients returning to Emilia Romagna from endemic areas. ${ }^{11}$ Considering that $A$. albopictus density is high in most of the region in the summer and that local Dengue transmission has been reported in a French region with a comparable environment, prevention of local outbreaks was one of the reasons for hospital admission of patients during viremia.

The four patients described above were part of a six-people cluster of serotype 3 Dengue virus infection. The clinical course was benign in all of the 6 cases ( 4 pediatrics and 2 adults) as expected in case of primary infection. However, even in the case of mild disease, hospitalization is helpful to monitor warning signs of potential complications, in addition to the prevention of transmission during viremia. ${ }^{12}$ Interestingly, laboratory data were $n$ accordance with those of the literature, reporting hyperferritinaemia in Dengue associated with thrombocytopenia and elevated liver enzymes. Hyperferritinaemia is considered a hallmark of diseases characterized by extensive immune activation (like haemophagocytic lymphohistiocytosis and macrophage activation syndrome) and in some studies is reported as a possible predictor for severe Dengue. ${ }^{13,14}$ Whereas all patients were infected with the same virus strain, the second couple of siblings (patients 3 and 4), had lower platelet counts, higher ferritinaemia and higher liver enzymes, as well as a slightly more severe clinical course. These observations may suggest a role of genetic/familiar susceptibility in immune response to viral infection, and hence in deermining the severity of clinical course. ${ }^{15}$

In our pediatric record, support therapy was necessary to treat symptoms (elevated temperatures, nausea, diarrhea).

Since no effective antiviral agents and validated vaccines are available, vector control is essential because the spreading of Aedes vectors is well documented also in non-endemic areas.

Parents advice (mosquito-preventive measures, education of caregivers to seek prompt medical care in case of a febrile illness)
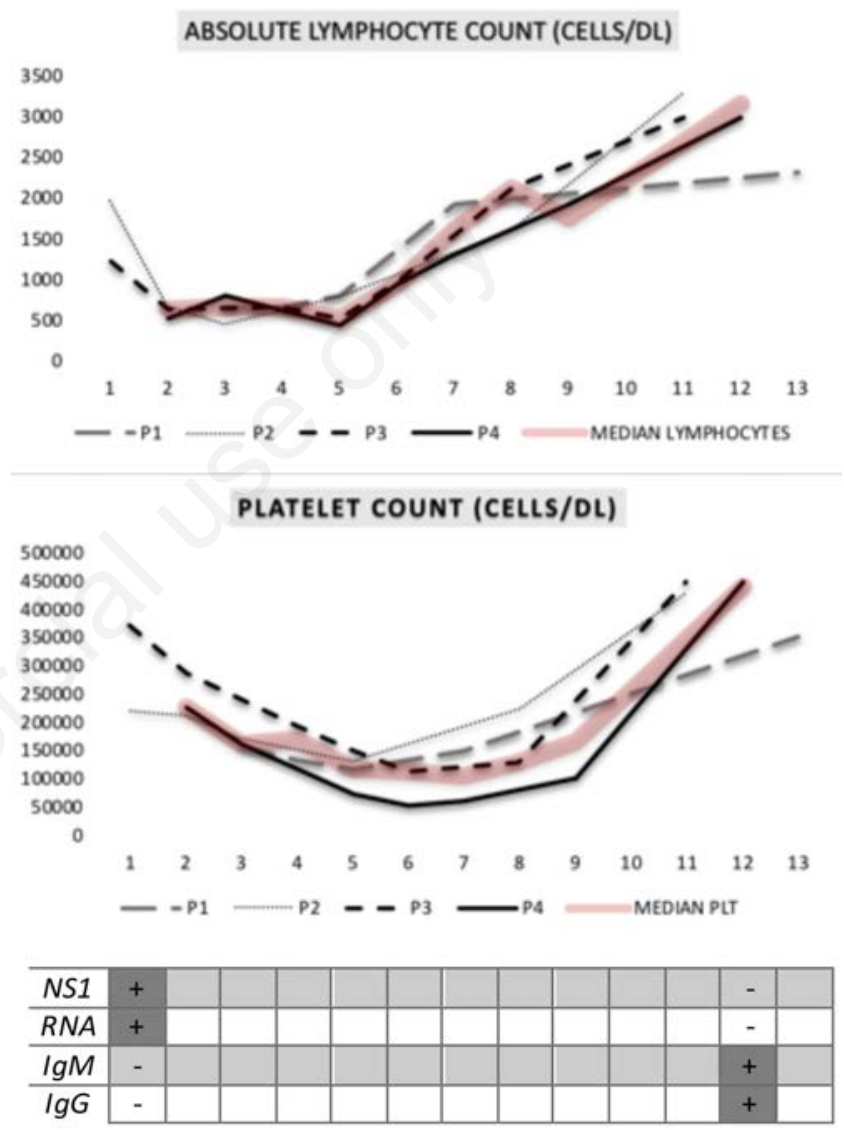

Figure 1. Median values of lymphocytes and platelets standardized against the time from fever onset and compared with the time of serological diagnosis and seroconversion.

Table 1. Main laboratoristic alterations reported. In brackets, days after the onset of fever with the maximum alteration.

\begin{tabular}{lcccc} 
& 1 & 2 & 3 & \\
WBC (N/dL) & $1630\left(4^{\text {th }}\right)$ & $1760\left(4^{\text {th }}\right)$ & $1680\left(3^{\text {rd }}\right)$ & 4 \\
Lymphocytes (N/dL) & $650\left(4^{\text {th }}\right)$ & $450\left(4^{\text {th }}\right)$ & $450\left(3^{\text {rd }}\right)$ & $1070\left(3^{\text {rd }}\right)$ \\
Neutrophils (N/dL) & $900\left(4^{\text {th }}\right)$ & $1500\left(4^{\text {th }}\right)$ & $530\left(4^{\text {th }}\right)$ & $230(1 s t)$ \\
Platelets (N/dL) & $118000\left(6^{\text {th }}\right)$ & $133000\left(6^{\text {th }}\right)$ & $51000\left(6^{\text {th }}\right)$ & $330\left(5^{\text {th }}\right)$ \\
\hline C-reactive protein (mg/dL) & $0.25\left(2^{\text {nd }}\right)$ & $1.39\left(1^{\text {st }}\right)$ & $1.3\left(1^{\text {st }}\right)$ & $110000\left(3^{\text {rd }}\right)$ \\
AST/ALT (UI/L) & $\left.68 / 77^{\text {th }}\right)$ & - & $2.01\left(4^{\text {th }}\right)$ & $1.14\left(2^{\text {nd }}\right)$ \\
aPTT & - & - & $1938\left(6^{\text {th }}\right)$ & $142 / 89\left(6^{\text {th }}\right)$ \\
FERRITIN (ng/mL) & $447\left(5^{\text {th }}\right)$ & $430\left(5^{\text {th }}\right)$ & $1.55\left(6^{\text {th }}\right)$ \\
\hline
\end{tabular}

WBC, white blood cells; N, number; AST, aspartate aminotransferase; ALT, alanine aminotransferase; aPTT, activated partial thromboplastin time. 
could lead to increased awareness of the risk of contracting Dengue when travelling to an endemic region.

\section{Conclusions}

Dengue should always be considered in the differential diagnosis of febrile children returning from endemic regions; this will allow early recognition and successful management. Diagnosis is based primarily on risk knowledge and the diffusion of rapid diagnostic tests (as NS1 rapid test) to early recognize all the cases, which should undergo careful observation for both clinical and epidemiological reasons. Even if, in non-endemic areas, the probability of facing a secondary infection and severe Dengue is low, awareness of a primary infection may help prevent re-exposure avoiding a reinfection with a potentially severe clinical course.

\section{References}

1. Guzman A, Istúriz RE. Update on the global spread of dengue. Int J Antimicrob Ag 2010;36:S40-2.

2. Simmons CP, Farrar JJ, van Vinh Chau N, Wills B. Dengue. N Engl J Med 2012;366:1423-32.

3. Verhagen LM, de Groot R. Dengue in children. J Infection 2014;69:S77-86.

4. World Health Organization. Dengue: guidelines for diagnosis, treatment, prevention and control: Chapter II. Geneva, Switzerland: WHO; 2009.

5. Jelinek T, Mühlberger N, Harms G, et al. Epidemiology and clinical features of imported dengue fever in Europe: sentinel surveillance data from TropNetEurop. Clin Infect Dis 2002;35:1047-52.
6. Schwartz E, Weld LH, Wilder-Smith A, et al.; GeoSentinel Surveillance Network. Seasonality, annual trends, and characteristics of dengue among ill returned travelers, 1997-2006. Emerg Infect Dis 2008;14:1081.

7. Fortuna $\mathrm{C}$, Remoli ME, Rizzo C, et al. Imported arboviral infections in Italy, July 2014-October 2015: a national reference laboratory report. BMC Infect Dis 2017;17:216.

8. International Center for Environment, Development and Operational Research (ENDEVOR) - Maldives. The National Environmental Health Action Plan (NEHAP: 2015-2020). 2014. Available from: http://www.searo.who.int/maldives/ mediacentre/nehap2015-2020.pdf?ua=1 Accessed: October 19, 2018.

9. Massad E, Amaku M, Coutinho FAB, et al. Estimating the probability of dengue virus introduction and secondary autochthonous cases in Europe. Sci Rep 2018;8:4629.

10. Rezza G, Nicoletti L, Angelini R, et al. Infection with chikungunya virus in Italy: an outbreak in a temperate region. Lancet 2007;370:1840-6.

11. Pierro A, Varani S, Rossini G, et al. Imported cases of dengue virus infection: Emilia-Romagna, Italy, 2010. Clin Microbiol Infect 2011;17:1349-52.

12. Kaushik A, Pineda C, Kest H. Diagnosis and management of dengue fever in children. Pediatr Rev 2010;31:e28-35.

13. Roy Chaudhuri S, Bhattacharya S, Chakraborty M, et al. Serum ferritin: a backstage weapon in diagnosis of dengue fever. Interdiscip Perspect Infect Dis 2017;2017:7463489.

14. Soundravally R, Agieshkumar B, Daisy M, et al. Ferritin levels predict severe Dengue. Infection 2015;43:13-9.

15. Van de Weg CA, Huits RM, Pannuti CS, et al. Hyperferritinaemia in dengue virus infected patients is associated with immune activation and coagulation disturbances. PLoS Negl Trop Dis 2014;8:e3214. 